\title{
CGH and CD 44/MIB-1 immunohistochemistry are helpful to distinguish metastasized from nonmetastasized sporadic pheochromocytomas
}

\author{
Christian August ${ }^{1}$, Kathrein August ${ }^{1}$, Soeren Schroeder ${ }^{2}$, Hannes Bahn $^{3}$, Raoul Hinze ${ }^{4}$, \\ Hideo A Baba ${ }^{5}$, Christian Kersting ${ }^{1}$ and Horst Buerger ${ }^{1, *}$ \\ ${ }^{1}$ Institute of Pathology, University Muenster, Germany; ${ }^{2}$ Institute of Immunology, Pathology and Molecular \\ Biology, Hamburg, Germany; ${ }^{3}$ Institute of Pathology, University Halle/S., Germany; ${ }^{4}$ Institute of Pathology, \\ Academic Hospital Schwerin, Germany and ${ }^{5}$ Institute of Pathology, University of Essen, Germany
}

\begin{abstract}
The natural course of pheochromocytomas (PCC) cannot be predicted for certain on the basis of primary histology, their malignant character can only be confirmed by the occurrence of metastases during follow-up. Based on the recently proposed PASS score for evaluation we examined 37 adrenal (36 sporadic and one familial) and six sporadic extra-adrenal paragangliomas (all designated as pheochromocytomas) with a 'malignant histology' to find additional predictive factors. Drawing upon the follow-up (18 months to 12 years, mean 5.8 years) metastasized $(n=20)$ and nonmetastasized $(n=23)$ courses could be distinguished. Metastasized PCC revealed significantly $(P=0.03)$ more copy number changes on comparative genomic hybridization (CGH) (mean 8.3) than nonmetastasized tumors (mean: 4.3). The most frequent chromosomal alterations were losses on $1 p(75.6 \%)$ and $3 q(44 \%)$. Both were detected with identical frequency in metastasized and nonmetastasized PCC. A gain on $17 q(P=0.025)$ was significantly predominant in malignant courses and suggests similarities in the genetic origin and progression of PCC and neuroblastomas. The proliferative activity (MIB-1 score) of metastasized PCC $(n=20)$ was found to be significantly higher in metastasized tumors (mean $12.8 \%$ vs mean $3.5 \%$ ). In contrast, the semiquantitatively scored membrane-bound staining of CD $44-S$ was stronger in tumors without metastases (mean 2.1 vs mean: 0.25$)$ during the follow-up period $(P<0.01)$. Although the results correspond to the established weight differences the tumor weight does not appear to be an independent prognostic factor. Our study suggests that CD 44-S and MIB-1 immunostaining as well as the CGH results might complement the PASS score in predicting a metastasized course of PCC. Regardless of tumor weight, tumors with a 'malignant histology' are highly prone to metastasize when more than $5 \%$ of MIB1positive nuclei are present or CD44-S immunostaining is negative, or both. PCC with 10 or more copy number changes on CGH must be referred to as malignant tumors.

Modern Pathology (2004) 17, 1119-1128, advance online publication, 28 May 2004; doi:10.1038/modpathol.3800160
\end{abstract}

Keywords: pheochromocytoma; predictive factors; CGH; immunohistochemistry; CD 44; MIB-1

The safe distinction between benign and malignant pheochromocytomas (PCC) is an unresolved dilemma beleaguering diagnostic pathology to the present day. In most cases the decision is therefore based on the occurrence of metastases as the only proof of malignancy. Recently, Thompson ${ }^{1}$ proposed an

Correspondence: Dr C August, Institute of Pathology, Münster University Hospital, Domagkstrasse 17, D-48149 Münster, Germany.

E-mail: AUGUSTC@uni-muenster.de

${ }^{*}$ Dedicated to Werner Boecker on his 60th birthday.

Received 18 September 2003; revised 25 January 2004; accepted 23 February 2004; published online 28 May 2004 adrenal gland scoring scale (PASS) capable of determining malignancy solely on the grounds of conventional histological criteria. Within the framework of this scoring scale, less prognostic importance is attributed to vascular and capsular invasion (with a score of 1 point each) than to growth pattern, necroses and characteristics of proliferative behavior (high cellularity, cellular monotony, more than three mitoses/10 HPF and the occurrence of atypical mitoses). These characteristics each have a score of two points).

In recent reports on adrenal and extra-adrenal PCC a number of chromosomal aberrations were discussed with a view to their possible involvement 
in tumor progression or malignant behavior. ${ }^{2-4}$ In those studies, the number of malignant adrenal PCC investigated was low (four, respectively, 10 sporadic malignant adrenal PCC) and the PASS scale had not yet been introduced.

Sporadic PCC are particularly suitable for analysis of chromosomal aberrations potentially important in tumor progression, because they are not affected by the well-established genetic aberrations of hereditary PCC that only occur extremely rarely in sporadic PCC. ${ }^{5-8}$ Moreover, the biological behavior of PCC has been analyzed in various immunohistochemical studies. ${ }^{9-12}$ Unlike the mitotic count, which was found to be useless for predicting the malignant behavior of PCC, ${ }^{13}$ the expression of Ki-67 is of prognostic significance. ${ }^{14,15}$ The expression of membrane-bound surface glycoprotein CD 44 in both the standard (S) form or its isoforms seems to be associated with invasive growth and development of metastases. ${ }^{16}$ However, the published results of CD 44 immunohistochemistry in various human tumors are too controversial to allow uniform predictive interpretation. ${ }^{17-20}$ Although CD 44 is associated with the degree of cellular differentiation in various endocrine tumors, if not with malignancy, ${ }^{21}$ membrane staining of CD 44 was described to be associated with a better outcome in neuroblastomas. ${ }^{22}$ Unfortunately, a systematic CD 44 immunohistochemical analysis of PCC with longterm follow-up has not yet been undertaken. This study focuses on PCC that were diagnosed as 'malignant' in primary histology and fulfilled the PASS criteria of malignancy after re-evaluation. The aim of this study was to explore the potential of cytogenetical or immunohistochemical methods to distinguish metastasized from nonmetastasized PCC with reference to an analysis of the follow-up. In such a way the purely conventional histology (PASS) might be reinforced with prognostic information of predictive value.

\section{Materials and methods}

\section{Light Microscopy and Immunohistochemistry}

Formalin-fixed and paraffin-embedded material from surgical specimens of 42 sporadic and one familial PCC (37 adrenal and six extra-adrenal) was investigated (Table 1). All tumors that had been classified as malignant PCC in primary histology were independently re-evaluated under light microscopy (hematoxylin and eosin (H\&E), PAS, E.v.G. stains) according to the PASS score proposed by Thompson ${ }^{1}$ by two investigators (CA; KA). Immunocytochemical staining was carried out using antisera against CD 44-S and CD 44-V6 (both Novocastra, monoclonal, microwave pretreatment, 1:50) and MIB-1 (DAKO, monoclonal, autoclave pretreatment, 1:50). Following the application of the bridging antibody against mouse the MIB-1 immunoreactivity was determined by peroxidase- antiperoxidase (PAP); CD 44-S and CD 44-V6 were determined using an ABC Kit (Vector Lab., Burlingame).

\section{Quantitative Evaluation of Immunostaining}

The membrane-bound immunostaining of CD 44-S was independently scored semiquantitatively by the above-mentioned investigators, using the following score: $(0=$ negative, $1+:<10 \%$ stained tumor cells, $2+: \quad 10-50 \%$ stained tumor cells, $3+:>50 \%$ stained tumor cells, $4+: 100 \%$ stained tumor cells). The nuclear MIB-1 immunostaining was evaluated semiquantitatively. The mean of stained nuclei of 10 high-power fields was correlated with the mean nuclear content in the same region. The results were rounded in that only increments of more than $5 \%$ were registered.

\section{Comparative Genomic Hybridization (CGH)}

In all, 41 tumors were analyzed by CGH, as described previously. ${ }^{23,24}$ DNA was isolated from paraffin sections $(10 \mu \mathrm{m})$ by proteinase $\mathrm{K}$ digestion followed by phenol-chloroform extraction according to standard protocols. Tumor-DNA was labeled with biotin-16-dUTP (Boehringer, Mannheim) and reference DNA from a healthy male donor was labeled with digoxigenin-11-dUTP (Boehringer, Mannheim) in a standard nick translation reaction. The DNAse I in the labeling reaction was adjusted in order to reveal an average fragment size of 500-1000 base pairs. Labeled DNA fragments were purified from remaining nucleotides by column chromatography (Sephadex G50).

Only metaphase spreads showing even highintensity hybridization with less granules were taken into account. Corresponding ratio profiles were evaluated only if the 95\% confidence limits did not exceed 0.15 . The $50 \%$ thresholds (upper threshold 1.25, lower threshold 0.75) were applied to define the chromosomal regions of DNA sequence losses or gains. Independent confirmation of chromosomal aberrations has shown that these thresholds are reliable and eliminate the possibility of false-positive results. ${ }^{24}$ The consistency of these aberrations has been confirmed by previous reverseCGH experiments (tumor DNA labeled with digoxigenin; reference DNA labeled with biotin). Each CGH experiment included a control hybridization of FITC and rhodamine labeled normal DNA to each other.

\section{Statistical Analysis}

The scored immunohistochemical results and the numeric results of CGH in correlation to the clinical follow-up were analyzed using the Mann-Whitney 
Table 1 Clinical dates and the gross pathological findings of all studied cases

\begin{tabular}{|c|c|c|c|c|c|c|c|c|c|}
\hline j-no & Age & Sex & Localization & Weight; size & PASS & Light microscopy & Metastases & Course & Follow-up period (Years) \\
\hline 1 & 17 & $\mathrm{f}$ & Extraadrenal & $817 \mathrm{~g} ; 15 \mathrm{~cm}$ & 8 & Angioinvasive & Vertebral column & Malignant & 4 \\
\hline 2 & 19 & $\mathrm{~m}$ & Extraadrenal & $1800 \mathrm{~g} ; 20 \mathrm{~cm}$ & 9 & Necroses & Liver & Malignant/died of disease & 3 \\
\hline 3 & 26 & $\mathrm{f}$ & Adrenal & Not available & 5 & & Liver, lungs & Malignant & 3 \\
\hline 4 & 27 & $\mathrm{~m}$ & Adrenal & $11 \mathrm{~g} ; 3.5 \mathrm{~cm}$ & 4 & & Not any & & 8 \\
\hline 5 & 29 & $\mathrm{~m}$ & Adrenal & $40 \mathrm{~g} ; 4.5 \mathrm{~cm}$ & 4 & & Not any & & 6 \\
\hline 6 & 29 & $\mathrm{f}$ & Adrenal & $42 \mathrm{~g} ; 5 \mathrm{~cm}$ & 5 & Angioinvasive, capsular invasion & Not any & & 6 \\
\hline 7 & 30 & $\mathrm{~m}$ & Adrenal & $82 \mathrm{~g} ; 7 \mathrm{~cm}$ & 6 & Capsular invasion & Not any & & 12 \\
\hline 8 & 31 & $\mathrm{~m}$ & Adrenal & $150 \mathrm{~g} ; 8.5 \mathrm{~cm}$ & 7 & Angioinvasive, capsular invasion & Not any & & 7 \\
\hline 9 & 31 & $\mathrm{f}$ & Adrenal & $46 \mathrm{~g} ; 4.5 \mathrm{~cm}$ & 5 & & Not any & & 3 \\
\hline 10 & 32 & $\mathrm{f}$ & Extraadrenal & Not available & 14 & Invasion of fat tissue & Metastases (nos) & Malignant & 2 \\
\hline 11 & 32 & $\mathrm{~m}$ & Extraadrenal & $1550 \mathrm{~g}$ & 6 & Ininfiltration of the kidney and v.cava & Vertebral column & Malignant & 2 \\
\hline 12 & 33 & $\mathrm{~m}$ & Extraadrenal & $22 \mathrm{~g} ; 4 \mathrm{~cm}$ & 8 & Invasion of the soft tissue & Lymphonodal & Malignant & 3 \\
\hline 13 & 34 & $\mathrm{f}$ & Extraadrenal & $303 \mathrm{~g} ; 11 \mathrm{~cm}$ & 11 & Necroses, invasion of v. cava & Metastases (nos) & Malignant & 2 \\
\hline 14 & 34 & $\mathrm{f}$ & Adrenal & $21 \mathrm{~g} ; 3.5 \mathrm{~cm}$ & 6 & & Not any & & 7 \\
\hline 15 & 35 & $\mathrm{~m}$ & Adrenal & $50 \mathrm{~g} ; 5 \mathrm{~cm}$ & 4 & & Not any & & 5 \\
\hline 16 & 36 & $\mathrm{~m}$ & Adrenal & $460 \mathrm{~g} ; 15 \mathrm{~cm}$ & 14 & Capsular invasive & Metastases (nos) & Malignant & 2 \\
\hline 17 & 38 & $\mathrm{f}$ & Adrenal & $68 \mathrm{~g}$ & 7 & Angioinvasive & Not any & & 12 \\
\hline 18 & 38 & $\mathrm{f}$ & Adrenal & $44 \mathrm{~g} ; 4 \mathrm{~cm}$ & 5 & & Not any & & 8 \\
\hline 19 & 38 & $\mathrm{~m}$ & Adrenal & $201 \mathrm{~g} ; 8.7 \mathrm{~cm}$ & 6 & & Not any & & 5 \\
\hline 20 & 39 & $\mathrm{f}$ & Adrenal & $82 \mathrm{~g} ; 6 \mathrm{~cm}$ & 12 & Angioinvasive & Lymphonodal & Malignant & 2 \\
\hline 21 & 41 & $\mathrm{~m}$ & Adrenal & $84 \mathrm{~g} ; 8 \mathrm{~cm}$ & 9 & Capsular invasive & Not any & & 12 \\
\hline 22 & 44 & $\mathrm{~m}$ & Adrenal & $180 \mathrm{~g} ; 11 \mathrm{~cm}$ & 15 & Necroses, invasive & Vertebral column & Malignant & 4 \\
\hline 23 & 45 & $\mathrm{~m}$ & Adrenal & $76 \mathrm{~g} ; 6 \mathrm{~cm}$ & 9 & Invasion of fat tissue & Not any & & 3 \\
\hline 24 & 47 & $\mathrm{~m}$ & Adrenal & $241 \mathrm{~g} ; 9 \mathrm{~cm}$ & 13 & Necroses, invasive & Metastases (nos) & Malignant & 3 \\
\hline 25 & 47 & $\mathrm{~m}$ & Adrenal & $199 \mathrm{~g} ; 8.5 \mathrm{~cm}$ & 6 & & Not any & & 10 \\
\hline 26 & 49 & $\mathrm{~m}$ & Adrenal & $37 \mathrm{~g} ; 4 \mathrm{~cm}$ & 6 & & Not any & & 8 \\
\hline 27 & 51 & $\mathrm{~m}$ & Adrenal & Not available & 7 & Invasion of fat tissue & Not any & & 9 \\
\hline 28 & 55 & $\mathrm{~m}$ & Adrenal & $270 \mathrm{~g} ; 11 \mathrm{~cm}$ & 10 & Angioinvasive & Metastases (nos) & Malignant/died of disease & 2 \\
\hline 29 & 56 & $\mathrm{f}$ & Adrenal & $173 \mathrm{~g} ; 8 \mathrm{~cm}$ & 4 & & Lymphonodal and Lungs & Malignant & 3 \\
\hline 30 & 58 & $\mathrm{f}$ & Adrenal & $38 \mathrm{~g} ; 4 \mathrm{~cm}$ & 5 & Angioinvasive & Not any & & 4 \\
\hline 31 & 58 & $\mathrm{f}$ & Adrenal & $3.7 \mathrm{~cm}$ & 8 & Capsular invasive & Not any & & 8 \\
\hline 32 & 59 & $\mathrm{f}$ & Adrenal & $70 \mathrm{~g} ; 6.5 \mathrm{~cm}$ & 5 & Angioinvasive & Lymphonodal & Malignant & 5 \\
\hline 33 & 60 & $\mathrm{f}$ & Adrenal & $67 \mathrm{~g} ; 6 \mathrm{~cm}$ & 13 & Necroses & Chest wall & Malignant & 2 \\
\hline 34 & 61 & $\mathrm{~m}$ & Adrenal & $350 \mathrm{~g}$ & 10 & Angioinvasive, capsular invasion & Metastases (nos) & Malignant & 2 \\
\hline 35 & 61 & $\mathrm{~m}$ & Adrenal & $45 \mathrm{~g} ; 5 \mathrm{~cm}$ & 14 & Angioinvasive, capsular invasion & Liver & Malignant & 3 \\
\hline 36 & 67 & $\mathrm{~m}$ & Adrenal & $64 \mathrm{~g} ; 6 \mathrm{~cm}$ & 8 & Capsular invasive & Not any & & 12 \\
\hline 37 & 67 & $\mathrm{~m}$ & Adrenal & $42 \mathrm{~g} ; 5 \mathrm{~cm}$ & 6 & Capsular invasive & Not any & & 12 \\
\hline 38 & 68 & $\mathrm{~m}$ & Adrenal & Not available & 8 & Angioinvasive, capsular invasion & Not any & & 11 \\
\hline 39 & 74 & $\mathrm{f}$ & Adrenal & Not available & 14 & Angioinvasive, capsular invasion & Metastases (nos) & Malignant/died of disease & 18 months \\
\hline 40 & 80 & $\mathrm{f}$ & Adrenal & $280 \mathrm{~g} ; 10 \mathrm{~cm}$ & 4 & Angioinvasive & Not any & & 8 \\
\hline 41 & 34 & $\mathrm{~m}$ & Adrenal & $146 \mathrm{~g} ; 6.5 \mathrm{~cm}$ & 11 & Angioinvasive & Lymphonodal & Malignant & 2 \\
\hline 42 & 50 & $\mathrm{~m}$ & Adrenal & $140 \mathrm{~g} ; 9.5 \mathrm{~cm}$ & 6 & Angioinvasive & Liver & Malignant & 2 \\
\hline $43^{*}$ & 37 & $\mathrm{~m}$ & Adrenal & $60 \mathrm{~g}$ and $28 \mathrm{~g}$ & 5 & Bilateral & Not any & & 3 \\
\hline
\end{tabular}

Abbreviations: $\mathrm{m}=$ male; $\mathrm{f}=$ female, nos $=$ not other specified

*This case (no. 43) was found to be a familial PCC (showing a ret 634 mutation). Metastasized courses are marked. 
$U$-test and Fisher's exact test. Probabilities of error lower than 0.05 were accepted as significant.

\section{Results}

\section{Clinical and Macroscopic Results}

The study included 43 patients (26 male and 17 female, aged between 17 and 80) with adrenal $(n=37)$ and extra-adrenal $(n=6)$ PCC. All 42 tumors had occurred sporadically without any association to hereditary syndromes. In one patient (Table 1, no 43) with bilateral PCC who had originally been reported to show no signs of hereditary pathogenesis, a ret 634 mutation according a MEN 2A syndrome was detected in the meantime. During the follow-up periods ranging from 18 months to 12 years (5.8 years). In all, 20 tumors developed metastases as opposed to 23 nonmetastasized cases. Clinical data including macroscopic findings is summarized in Table 1. All six extra-adrenal PCC showed metastatic spread. The occurrence of metastases in the follow-up period was related to tumor weight. Ranging from 11 to $1800 \mathrm{~g}$, the tumor weight of metastasized PCC (mean: $395 \mathrm{~g}$ ) was significantly higher than that of nonmetastasized tumors (mean: $75 \mathrm{~g}, P<0.05)$. Of the metastasized PCC $75 \%$ weighed more than $100 \mathrm{~g}$. Tumors whose weight amounted to or exceeded $100 \mathrm{~g}$ were significantly more prone to metastasize $(P<0.05)$.

\section{Light Microscopy}

All tumors including those with extra-adrenal localization were examined using the PASS-score. Vascular or capsular invasion and nuclear pleomorphism scored one point, growth pattern, cellularity, mitoses counts exceeding 3/10 HPF and infiltration of adipose tissue scored two points according to Thompson. ${ }^{1}$ The results are demonstrated in Table 1. Tumors which upon re-evaluation had a score of at least four points were regarded as malignant. More than $90 \%$ of all tumors showed a characteristic pattern with solid nests of cells (Figure 1a). The remaining PCC were solid tumors
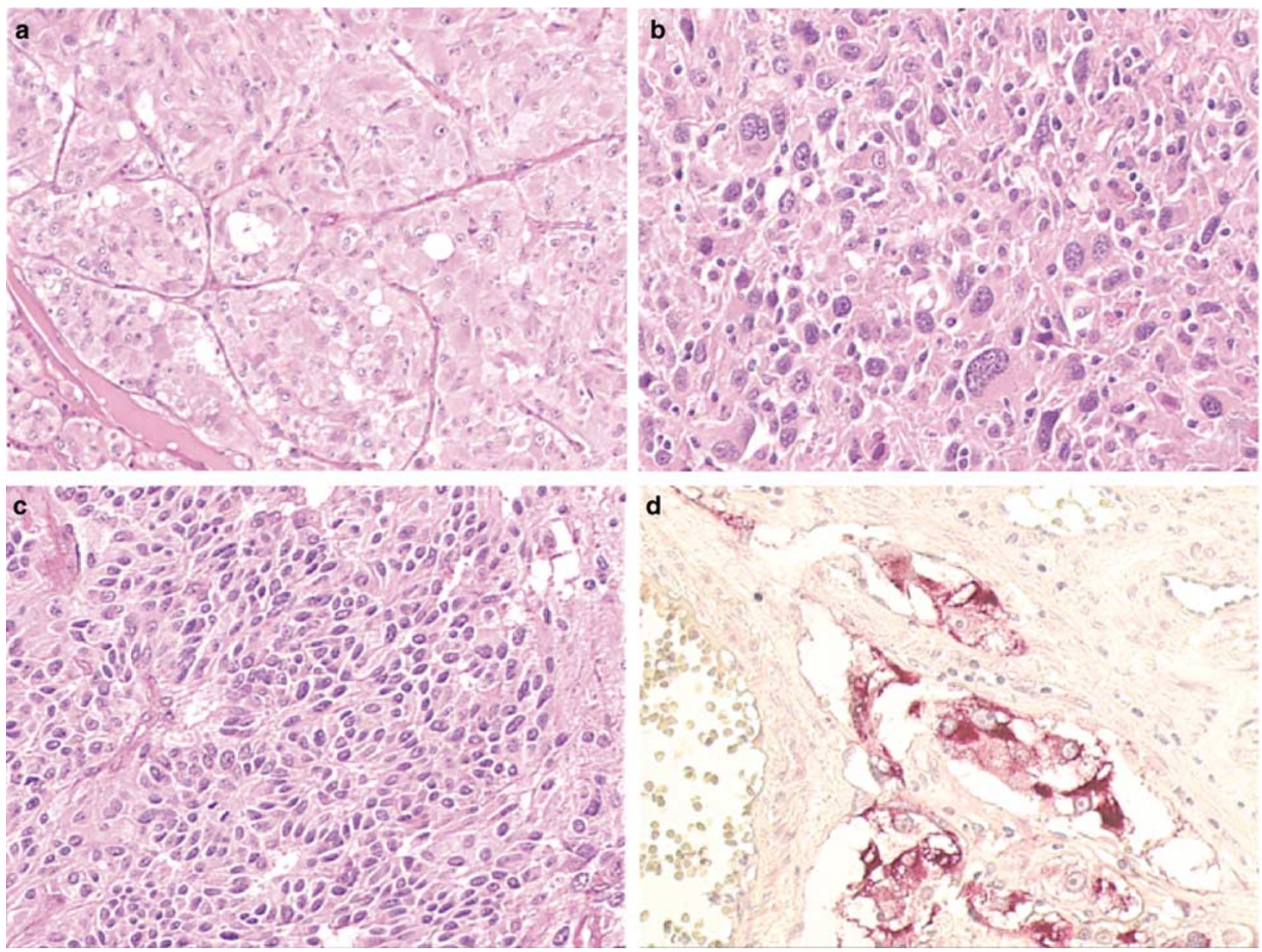

Figure 1 Light microscopy of pheochromocytomas: (a) solid pattern ('classic' type, H\&E, $\times 100$ ); (b) irregular growth pattern with nuclear polymorphism (H\&E, $\times 250)$; (c) neuroblastoma-like pattern of pheochromocytoma (H\&E, $\times 100)$; (d) capsular infiltration and angioinvasiveness (immunostaining with Chromogranin A, × 250). 
with in some cases marked nuclear irregularities (Figure 1b). A neuroid or neuroblastoma-like pattern (Figure 1c) was only rarely found. Tumors of both groups frequently showed blunt margins and invasive growth into the vessels and capsule (Figure 1d).

\section{Comparative Genomic Hybridization}

In all, 41 tumors were examined by CGH. In two cases the isolation of DNA could not be accomplished. Chromosomal imbalances were detected in 39 cases. Two PCC revealed no evidence of the presence of unbalanced chromosomal alterations. The number of genetic alterations ranged from 0 to

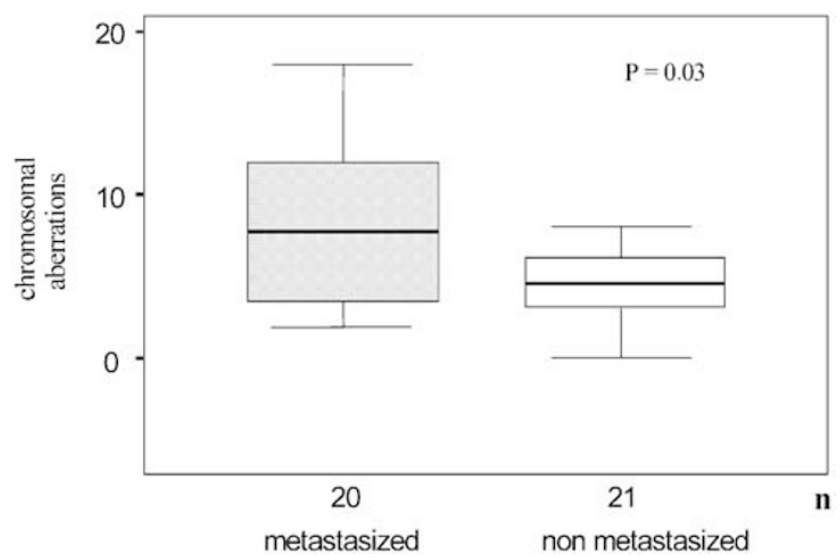

Figure 2 Molecular cytogenetic results in CGH $(n=41)$ : Metastasized courses showed significantly more unbalanced numeric chromosomal aberrations (mean 8.3) that nonmetastasized ones (mean 4.3). The median is shown as a dark line.
18. The most frequent losses were found on chromosome arms $1 \mathrm{p}(75.6 \%)$ and $3 \mathrm{q}$ (44\%; Figure 3 ) followed by losses of 22q (29.2\%), 11q (22\%), 6q $(19.5 \%), 11 p(17 \%)$ and $21 q(17 \%)$. Gains were observed predominantly on 17q (36.6\%), 20q (22\%), $7 p, 9 q$ and $12 q$ (all 14.6\%). Among extra-adrenal PCC, chromosomal losses on $1 p(4 / 6), 1 q(3 / 6) 6 q$ $(2 / 6)$ and $11 p(2 / 6)$ were most frequent.

\section{CGH Alterations in PCC with Benign and Malignant Courses}

Metastasized tumors showed significantly $(P=0.03)$. more genetic alterations (range: 2-18; mean 8.3) than cases without any occurrence of metastases (range: 0-8; mean 4.3) during the followup (Figure 2). PCC with 10 chromosomal aberrations or more $(n=8)$ developed metastases without any exception $(100 \%$ of cases). PCC with more than eight genetic alterations $(n=14)$ showed metastasized courses in $85.7 \%$.

An overview of the frequency of numeric chromosomal gains and losses and their distribution between both groups of the biologically divergent follow-up is shown in Figure 3. Losses on $1 p$ and $3 q$ were found to be the most frequent numeric chromosomal alterations. They were evenly distributed between metastasized and nonmetastasized PCC. Equally, losses of 8p, 11p, 11q, and 22q occurring in $15 \%$ to nearly $30 \%$ of the cases showed no significant prevalence in metastasized courses. The loss of $9 p$ in $9.7 \%$ of cases (see Table 2) was found exclusively in metastasized courses $(P=0.048)$. 
Table 2 Immunohistochemcial results and the number of chromosomal aberrations detected by CGH are shown

\begin{tabular}{|c|c|c|c|c|c|c|c|c|c|c|c|c|c|c|c|c|c|c|c|c|c|c|c|c|c|}
\hline Case no. & $C D 44$ & $\% M I B-1$ & $\mathrm{CGH}$ & $1 p$ & $1 q$ & $3 p$ & $3 q$ & $6 p$ & $6 q$ & $7 p$ & $7 q$ & $8 p$ & $8 q$ & $9 p$ & $9 q$ & $11 p$ & $11 q$ & $12 p$ & $12 q$ & $17 p$ & $17 q$ & $20 p$ & $20 q$ & $21 q$ & $22 q$ \\
\hline 1 & 0 & 5 & 3 & 0 & 1 & 0 & 0 & & & & & & & & & & & & & & & & & & \\
\hline 2 & 0 & 5 & 2 & 0 & & 0 & 0 & & & & & & & & & & & & & & & & & & \\
\hline 3 & 0 & 5 & 10 & 0 & 1 & 0 & 0 & & & 1 & & 0 & 0 & & & & & 0 & & & 1 & & & & 0 \\
\hline 4 & 0 & 1 & 0 & & & & & & & & & & & & & & & & & & & & & & \\
\hline 5 & 2 & 1 & 0 & & & & & & & & & & & & & & & & & & & & & & \\
\hline 6 & 2 & 5 & 7 & 0 & 1 & 0 & 0 & & & & & & & & & & & & & 1 & 1 & & 1 & 0 & 0 \\
\hline 7 & 1 & 1 & 5 & 0 & & & & 1 & 0 & & & & & & & & & & & 0 & & & & & \\
\hline 8 & $(+)$ & 5 & 1 & 0 & & & & & & & & & & & & & & & & & & & & & \\
\hline 9 & 2 & 5 & 3 & 0 & & & 0 & & & & & & & & & 0 & 0 & & & & & & & & \\
\hline 10 & 0 & 5 & 10 & & & & 0 & & 0 & & 0 & & & & 1 & & 0 & & & & 1 & & 1 & & \\
\hline 11 & $(+)$ & 5 & 4 & 0 & & & & & & & & & & & & 0 & & & 1 & & & & 1 & & \\
\hline 12 & 2 & 5 & 2 & 0 & & & & & & & & & & & & 0 & & & & & & & & & \\
\hline 13 & $(+)$ & 5 & 8 & 1 & & & & & 0 & & & & & & & & & & & 1 & 1 & & 1 & & 1 \\
\hline 14 & 0 & 5 & $\mathrm{n}$ & & & & & & & & & & & & & & & & & & & & & & \\
\hline 15 & 2 & 5 & 8 & 0 & 1 & 0 & 0 & & & & & & & & & & & & & 0 & 1 & & & & 0 \\
\hline 16 & 0 & 20 & 16 & 0 & 0 & & 0 & & & & & 0 & 0 & 0 & 0 & & 1 & 1 & 1 & & & 1 & 1 & 1 & \\
\hline 17 & 1 & 1 & 5 & 0 & & & 0 & & & & & 0 & 0 & & & & & & & & 1 & & & & 0 \\
\hline 18 & 3 & 5 & 4 & & & & & & & 1 & 1 & 1 & 1 & & & 0 & 0 & & & & & 1 & 1 & & \\
\hline 19 & $(+)$ & 5 & 5 & 0 & & & & 1 & 0 & & & & 1 & & & 1 & & & & & & & & & 0 \\
\hline 20 & 0 & 5 & 2 & & & & & & & & & & & & & & & & & & & & & 0 & \\
\hline 21 & 0 & 0 & 6 & 0 & & & 0 & & & & & & & & & & 0 & & & 0 & 0 & & & & 0 \\
\hline 22 & 0 & 30 & 10 & 0 & & 0 & & 1 & & & & & & & 1 & & 0 & & & 0 & 1 & & & & 0 \\
\hline 23 & 1 & 1 & 3 & & & & & & & & & & & & & & & & & & & & & 0 & \\
\hline 24 & 0 & 10 & 14 & 0 & 1 & & 0 & & 1 & 1 & & & & & & 0 & 0 & 1 & 1 & & 1 & & & 0 & 1 \\
\hline 25 & 2 & 5 & 2 & 0 & & & & & & & & & & & & & & & & & & & & & 1 \\
\hline 26 & $(+)$ & 1 & 1 & 0 & & & & & & & & & & & & & & & & & & & & & \\
\hline 27 & 2 & 1 & 6 & 0 & & & & 1 & 0 & & & & & & 1 & 1 & & & & & 1 & & & 0 & \\
\hline 28 & 0 & 10 & 5 & 0 & & & & & & & & & & & & & & 0 & & & & & & & \\
\hline 29 & 0 & 5 & 8 & 0 & 1 & & & & & & 0 & 0 & 0 & 0 & & & 0 & & & 1 & 1 & & & & \\
\hline 30 & 2 & 5 & 5 & 0 & & 0 & 0 & & 0 & & & & & & & & & & & 0 & & & & & 0 \\
\hline 31 & 3 & 5 & $\mathrm{n}$ & & & & & & & & & & & & & & & & & & & & & & \\
\hline 32 & 0 & 20 & 9 & 0 & & & 0 & 0 & 1 & & & & 0 & & & 0 & & & & 0 & & & & & \\
\hline 33 & 0 & 40 & 17 & 0 & 1 & & & & & 1 & 1 & & 1 & 0 & 1 & 1 & & 1 & 1 & & 1 & 1 & 1 & & \\
\hline 34 & 0 & 5 & 18 & 0 & 0 & & 0 & & 1 & 1 & 0 & & & & 1 & 0 & 0 & & 1 & & 1 & & & 1 & \\
\hline 35 & 0 & 15 & 5 & & & & & & & & & 1 & 1 & & & & & & 1 & & 1 & 1 & 1 & & \\
\hline 36 & 1 & 5 & 3 & 0 & & & & & & & & & & & & 1 & & & & & & & & & 0 \\
\hline 37 & $(+)$ & 1 & 7 & 0 & & & 0 & & 0 & & & & & & 1 & & 0 & & & 0 & 0 & & & & 0 \\
\hline 38 & 1 & 5 & 6 & 0 & 1 & & 0 & & & & & 0 & & & & & & & & & & & & & 0 \\
\hline 39 & 0 & 50 & 15 & & & & & & & 1 & 1 & 0 & & 0 & & & & 1 & & 1 & 1 & & 1 & & 1 \\
\hline 40 & $(+)$ & 10 & 4 & 0 & & & & & & & 0 & & & & & & & 0 & & & & & & & \\
\hline 41 & 0 & 10 & 3 & & & & & & & & 1 & 1 & & & & & & & & & & & & & 1 \\
\hline 42 & 0 & 1 & 5 & 0 & & & 0 & & & & & & & & & & & & & & 1 & & & 0 & \\
\hline $43^{*}$ & 3 & 5 & 8 & 0 & & 0 & 0 & & 0 & & & & & & 0 & & & & & 0 & & & & 0 & 0 \\
\hline
\end{tabular}

The CD 44-S immunohistochemistry is demonstrated with reference to the score explained above. Metastasized courses are illuminated.

Gains of genetic material that were significantly more frequently associated with malignant courses were found on $17 \mathrm{q}(P=0.025) 12 \mathrm{q}(P=0.009)$ and 20q (0.048). Gains on 7p, 9q, 12p and $12 q$ were observed predominantly in tumors displaying 10 or more copy number changes.

\section{Immunohistochemistry}

Immunohistochemical findings are also summarized in Table 2. Significant differences in nuclear MIB-1 staining (Figures 4 and 5) between metastasized (range: 6.7-19.0, mean: 12.8) and nonmetastasized PCC (range: 3.3-4.6; mean 3.5) were observed $(P=0.015)$. The presence of more than $5 \%$ MIB-1stained nuclei were associated with malignant courses in $85 \%$ of cases (Figure 5). A total of $25 \%$ of metastasized PCC contained more than 10\% positive nuclei. All nonmetastasized PCC revealed less than 5\% MIB-1-stained nuclei.

CD 44-S reactivity was only recorded as positive in the event of a membrane-bound pattern of immunostaining. Immunoreactivity with CD 44-S (Figures 4d and e and 6) was significantly higher in nonmetastasized PCC (range: $0-4$; mean 2.1) than in metastasized courses (range: 0-3, mean: 0.25) during the follow-up period $(P<0.01)$.

There was no detectable reactivity with antibodies against CD 44-V6.

\section{Correlation between Immunohistochemical and Cytogenetic Results}

All eight tumors displaying more than 10 chromosomal aberrations (average weight: $259.6 \mathrm{~g}$ ). were 

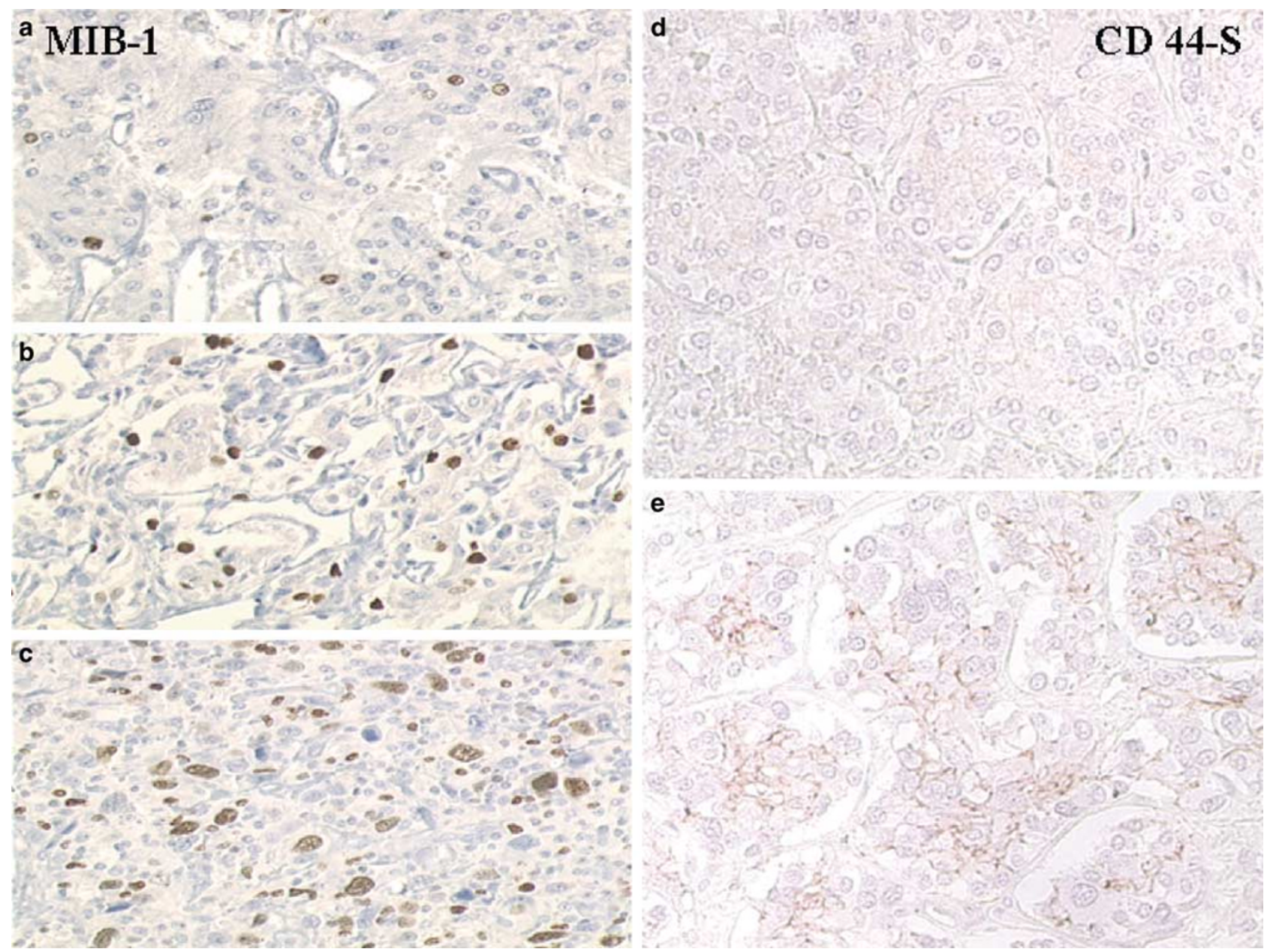

Figure 4 Immunohistochemistry of pheochromocytomas: left side: MIB-1 immunostaining, (a) approximately 1\%; (b) < 5\%; (c) 10-20\% stained nuclei; right side: CD 44-S immunostaining: (d) absence of CD 44-S immunostaining; (e) membrane-bound staining of CD 44-S.

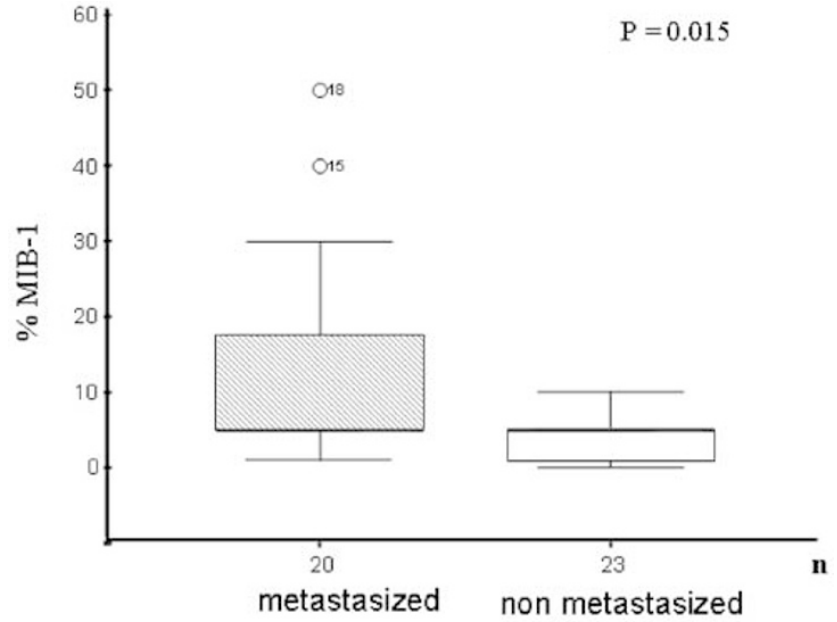

Figure 5 Semiquantitativelyl scored MIB-1 reactivity showing a significant difference in metastasized and nonmetastasized PCC (mean $12.8 \%$ vs $3.5 \%$ ). The median is shown as a dark line.

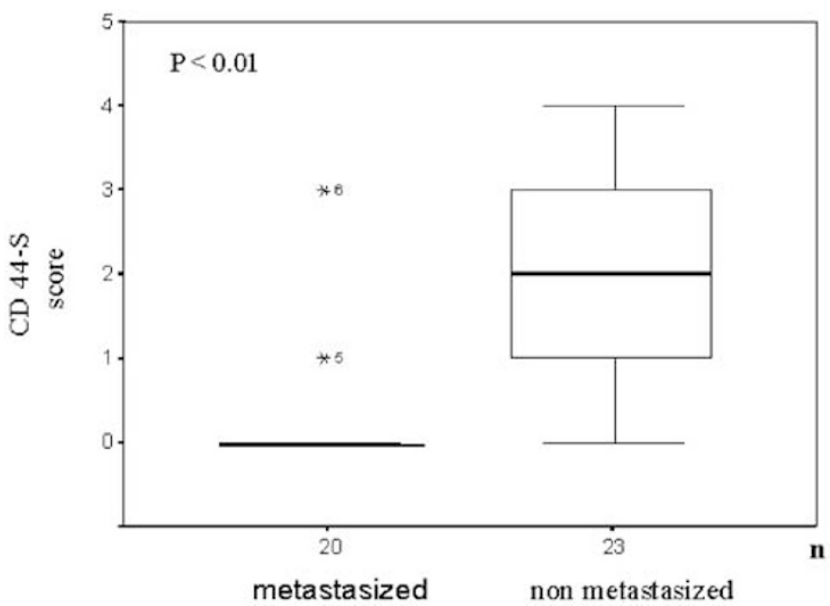

Figure 6 Semiquantitatively scored CD 44-S staining with difference between both studied groups (mean 2.1 vs 0.25 ). The median is shown as a dark line. 
characterized by completely negative reactions with antibodies against CD $44-\mathrm{S}$. $62.5 \%$ of these tumors showed more than $10 \%$ of MIB-1-stained nuclei.

\section{Discussion}

This study evaluates the relationship between several clinical, histological, immunohistochemical and cytogenetic features of PCC with the aim of predicting the development of metastases more accurately. To this end a well-defined series of 43 PCC with a 'malignant histology' according to the PASS score was investigated. ${ }^{1}$ One case included in the series has in the meantime been identified as MEN 2A associated. All other tumors can be defined as sporadic ones without any signs of hereditary disease. In all, 20 patients developed metastases during the follow-up (range 18 months to 12 years, average 5.8 years). The aim of our study was to distinguish tumors of both groups, although it cannot be ruled out that patients as yet without metastases may develop them at a later stage.

In this series metastasized and nonmetastasized PCC can be easily distinguished on the grounds of tumor weight. Tumors whose weight amounted to or exceeded $100 \mathrm{~g}$ showed metastases with significantly greater frequency $(P<0.05)$. Of metastasized PCC $75 \%$ weighed more than $100 \mathrm{~g}$. Nevertheless, the threshold of $100 \mathrm{~g}$ was not defined as a cutoff point in metastasized courses because there is a large overlap with malignant lesions as small as $22 \mathrm{~g}$ and benign ones that weighed $280 \mathrm{~g}$. This fact supports the generally accepted observation that tumor weight is not an independent prognostic marker in PCC. ${ }^{1}$

The most important aim of this study was the characterization of chromosomal aberrations on CGH and their correlation to clinical courses. Metastasized tumors showed significantly more copy number changes than tumors that did not develop any metastases during the observed followup (8.3 vs 4.3). Losses of $1 p$ and $3 q$ recently detected in PCC $^{2,3}$ were the most frequent chromosomal alterations in our study. They occurred in both groups with similar frequency. Therefore, they may be indicative early events in the tumorigenesis of sporadic PCC. However, these lesions probably are of less importance in view of progression to malignancy.

In neuroblastomas as the classical adreno-medullary tumor of childhood, a patient-tailored prognosis and treatment can be made based on genetic features. ${ }^{25}$ Our PCC results were similar to neuroblastoma with a prevalence of losses on $1 p$ and gains on the distal end of $17 q$. The gains of $17 q$ never reported before in PCC emerged as the most important genetic result in our study. We found a significant correlation between gains of $17 q$ and the occurrence of metastases in PCC. This was further confirmed in recent studies of neuroblastoma according to which accumulation of genetic material on $17 \mathrm{q}$ is associated with an adverse outcome. ${ }^{26,27}$ Losses of $1 \mathrm{p}$ were not only found in adrenomedullary but also in various other solid human tumors of adreno-cortical ${ }^{28}$ or other origins. ${ }^{29}$ Changes on the short arm of chromosome 1, that might be important in sporadic and hereditary PCC, were localized investigating restriction length fragments. These revealed losses of heterozygosity in $1 \mathrm{p} 31-36^{2,30,31}$ which might be indicative of an inactivation of tumor suppressor genes in this segment. The suppressor gene p73 on $1 p 36^{32}$ which in neuroblastomas is clearly associated with a poor prognosis ${ }^{27}$ seems to be of lesser importance in PCC. ${ }^{2}$

In this study, gains on $12 q(6 / 0)$ and $20 q(7 / 2)$ and losses of $9 p(4 / 0)$ were found in $10-22 \%$ of all cases with a statistical predominance in metastasized tumors. However, these results are not strong enough to support a definite correlation and need to be confirmed by further investigations.

In contrast losses of $8 p, 11 p, 11 q$ and $22 q$ that were identified with a frequency ranging from $15 \%$ to nearly $30 \%$ were not statistically associated with metastasized spread.

Our results do not confirm that alterations on chromosome 11 are of particular importance for distinguishing between metastasized and nonmetastasized courses, as suggested assumed by Edström et $a l^{2}$ In our study, losses of 11p and 11q occurred with identical frequency in both groups of PCC. Moreover, our results did not confirm the hypothesis of Dannenberg et $a l^{3}$ that a loss of $6 \mathrm{q}$ is an important event in tumor progression. In our study, losses of $6 q$ were predominantly associated with nonmetastasized tumors. A loss of $17 p$ where the p53 gene is localized was only detected in six (6/8) of nonmetastasized and two (2/8) of metastasized PCC.

In this series, the presence of 10 and more copy number changes within a single tumor was always associated with the development of metastases at a later stage. The presence of eight chromosomal aberrations was associated with the occurrence of metastases in $85 \%$ of cases. Since $60 \%$ of the metastasized tumors that weighted less than $100 \mathrm{~g}$ showed less than six chromosomal aberrations on $\mathrm{CGH}$, we assume that genetic instability linked to tumor size and weight may be a sign of tumor progression, however, tumor progression may also be influenced by other mechanisms. Therefore, the occurrence of less than five chromosomal aberrations does not necessarily preclude development of metastases at a later stage.

Our results demonstrate the value of MIB-1 immunostaining as a tool for distinguishing between metastasized and nonmetastasized PCC independent of their respective weight. The metastasized tumors generally showed a proliferative activity of more than 5\%. All metastasized PCC that weighed less than $100 \mathrm{~g}$ revealed at least $5 \%$ of MIB-1-stained nuclei. The proliferative activity seems to be of 
prognostic significance irrespective of tumor weight. In other studies a lower mean MIB-1 index threshold of $2.5 \%$ of stained nuclei between benign and malignant PCC was reported. ${ }^{14}$

CD 44 is a cell surface glycoprotein, that mediates adhesive properties and junctions of various cells. Our study clearly demonstrates the beneficial importance of membrane-bound CD 44-S in PCC (Figure 6). In contrast, the absence of CD 44-S was significantly associated with metastasized courses even in those tumors weighing less than $100 \mathrm{~g}$. These findings corroborated similar results for neuroblastomas according to which CD 44 expression is associated with a better prognosis. ${ }^{22}$ Tumors deriving from the neural crest in general appear to be influenced by expression of CD 44-S. Following an aggravation of cohesive features among tumor cells their potential to generate metastases may decrease with expression CD 44-S on the cell surface. Interestingly, expression of CD 44-V6 was not reliably confirmed in this series.

In conclusion, numeric chromosomal aberrations on CGH and immunohistochemistry of CD 44-S and MIB-1 enable predicting the likelihood of metastases in PCC with 'malignant histology' with greater accuracy, if they are performed in addition to the conventional work-up of tumors according the PASS score. MIB-1 and CD44-S immunohistochemistry are techniques that can be easily applied. CD 44-Snegative PCC with more than $5 \%$ of MIB- 1 stained nuclei and/or tumors with 10 or more chromosomal aberrations on CGH are prime candidates for later developing metastases, regardless of tumor weight.

The chromosomal gain of $17 \mathrm{q}$ is an additional cytogenetic marker that in this study emerged as an important indicator of the biological behavior of PCC and suggests similarities with the genetic pathways of tumor progression in neuroblastoma. Further studies are required to define genetic markers for prognostic purposes in PCC. It will therefore be necessary to narrow down the critical chromosomal regions and to characterize the putative genes involved in tumor progression of sporadic PCC.

\section{Acknowledgements}

We thank Lydia Grote, Ulrike Neubert and Bettina. Brömmelkamp of the Institute of Pathology, Muenster University Hospital, for their excellent technical assistance.

\section{References}

1 Thompson LDR. Pheochromocytoma of the adrenal gland scaled score PASS to separate benign from malignant neoplasms. Am J Surg Pathol 2002;26: $551-566$.
2 Edstrom E, Mahlamaki E, Nord B, et al. Comparative genomic hybridization reveals frequent losses of chromosomes $1 \mathrm{p}$ and $3 \mathrm{q}$ in pheochromocytomas and abdominal paragangliomas, suggesting a common genetic etiology. Am J Pathol 2000;156:651-659.

3 Dannenberg H, Speel EJ, Zhao J, et al. Losses of chromosomes $1 p$ and $3 q$ are early genetic events in the development of sporadic pheochromocytomas. Am J Pathol 2000;157:353-359.

4 Koch CA, Vortmeyer AO, Huang SC, et al. Genetic aspects of pheochromocytoma (Review). Endocr Regul 2001;35:43-52.

5 Rodien P, Jeunemaitre X, Dumont C, et al. Genetic alterations of the RET proto-oncogene in familial and sporadic pheochromocytomas. Horm Res 1997;47: 263-268.

6 Eng C, Crossey PA, Mulligan LM, et al. Mutations in the RET proto-oncogene and the von Hippel-Lindau disease tumour suppressor gene in sporadic and syndromic phaeochromocytomas. J Med Genet 1995;32: 934-937.

7 Arnold A. RET mutation screening in sporadic pheochromocytoma. J Clin Endocrinol Metab 1996;81:430.

8 Bar M, Friedman E, Jakobovitz O, et al. Sporadic phaeochromocytomas are rarely associated with germline mutations in the von Hippel-Lindau and RET genes. Clin Endocrinol (Oxf) 1997;47:707-712.

9 Gupta D, Shidham V, Holden J, et al. Prognostic value of immunohistochemical expression of topoisomerase alpha II, MIB-1, p53, E-cadherin, retinoblastoma gene protein product, and HER-2/neu in adrenal and extraadrenal pheochromocytomas. Appl Immunohistochem Mol Morphol 2000;8:267-274.

10 de Krijger RR, van der Harst E, van der Ham F, et al. Prognostic value of p53, bcl-2, and c-erbB-2 protein expression in phaeochromocytomas. J Pathol 1999;188: 51-55.

11 Achilles E, Padberg BC, Holl K, et al. Immunocytochemistry of paragangliomas-value of staining for S-100 protein and glial fibrillary acid protein in diagnosis and prognosis. Histopathology 1991;18: 453-458.

12 Clarke MR, Weyant RJ, Watson CG, et al. Prognostic markers in pheochromocytoma. Hum Pathol 1998;29: 522-526.

13 Linnoila RI, Keiser HR, Steinberg SM, et al. Histopathology of benign versus malignant sympathoadrenal paragangliomas : clinicopathologic study of 120 cases including unusual histologic features. Hum Pathol 1990;21:1168-1180.

14 van der Harst E, Bruining $\mathrm{HA}$, Jaap $\mathrm{BH}$, et al. Proliferative index in phaeochromocytomas: does it predict the occurrence of metastases? J Pathol 2000;191:175-180.

15 Nagura S, Katoh R, Kawaoi A, et al. Immunohistochemical estimations of growth activity to predict biological behavior of pheochromocytomas. Mod Pathol 1999;12:1107-1111.

16 Barshack I, Goldberg I, Nass D, et al. CD44 expression in normal adrenal tissue and adrenal tumours. J Clin Pathol 1998;51:52-54.

17 Ross JS, Sheehan CE, Williams SS, et al. Decreased CD44 standard form expression correlates with prognostic variables in ovarian carcinomas. Am J Clin Pathol 2001;116:122-128.

18 Ayhan A, Tok EC, Bildirici I. Overexpression of CD44 variant 6 in human endometrial cancer and its 
prognostic significance. Gynecol Oncol 2001;80: 355-358.

19 Nguyen VN, Mirejovsky T, Melinova L, et al. CD44 and its v6 spliced variant in lung carcinomas: relation to NCAM, CEA, EMA and UP1 and prognostic significance. Neoplasma 2000;47:400-408.

20 Daniel L, Lechevallier E, Giorgi R, et al. CD44s and CD44v6 expression in localized T1-T2 conventional renal cell carcinomas. J Pathol 2001;193:345-349.

21 Komminoth P, Seelentag WK, Saremaslani P, et al. CD44 isoform expression in the diffuse neuroendocrine system II Benign and malignant tumors. Histochem Cell Biol 1996;106:551-562.

22 Terpe HJ, Christiansen H, Gonzalez M, et al. Differentiation and prognosis of neuroblastoma in correlation to the expression of CD44s. Eur J Cancer 1995;31A:549-552.

23 Kalloniemi A, Kalloniemi OP, Sudar D, et al. Comparative genomic hybridization for molecular cytogenetic analysis of solid tumors. Am J Surg Pathol 1992;258:818-821.

24 Buerger H, Otterbach F, Simon R, et al. Different genetic pathways in the evolution of invasive breast cancer are associated with distinct morphological subtypes. J Pathol 1999;189:521-526.

25 Brinkschmidt C, Christiansen H, Terpe HJ, et al. Comparative genomic hybridization (CGH) analysis of neuroblastomas-an important methodological approach in paediatric tumour pathology. J Pathol 1997;181:394-400.
26 Brinkschmidt C, Christiansen H, Terpe HJ, et al. Distal chromosome 17 gains in neuroblastomas detected by comparative genomic hybridization (CGH) are associated with a poor clinical outcome. Med Pediatr Oncol 2001;36:11-13.

27 Bown N, Cotterill S, Lastowska M, et al. Gain of chromosome arm 17q and adverse outcome in patients with neuroblastoma. N Engl J Med 1999;340: 1954-1961.

28 Zhao J, Speel E, Muletta-Feurer S, et al. Analysis of genomic alterations insporadic adrenocortical lesions: gains of chromosome 17 is an early event in adrenocortical tumorigenesis. Am J Pathol 1999;155: 1039-1045.

29 Ragnarsson G, Eiriksdottir G, Johannsdottir JT, et al. Loss of heterozygosity at chromosome 1p in different solid human tumours: association with survival. Br J Cancer 1999;79:1468-1474.

30 Vargas MP, Zhuang Z, Wang C, et al. Loss of heterozygosity on the short arm of chromosomes 1 and 3 in sporadic pheochromocytoma and extraadrenal paraganglioma. Hum Pathol 1997;28: 411-415.

31 Benn DE, Dwight T, Richardson AL, et al. Sporadic and familial pheochromocytomas are associated with loss of at least two discrete intervals on chromosome $1 \mathrm{p}$. Cancer Res 2000;60:7048-7051.

32 Kaghad M, Bonnet H, Yang A, et al. Monoallelically expressed gene related to p53 at 1p36, a region frequently deleted in neuroblastoma and other human cancers. Cell 1997;90:809-819. 